\title{
Monitoring Parkinson's Disease Progression Using Behavioural Inferences, Mobile Devices and Web Technologies
}

\author{
Interaction Analysis and Modelling Lab \\ University of Manchester \\ WWW'16 Conference. PhD Symposium
}

\author{
Julio Vega
}

Supervisors: Simon Harper, Caroline Jay \& Markel Vigo 


\section{Parkinson's Disease (PD)}

$\rightarrow$ Chronic neurodegenerative disease

$\rightarrow 1$ in 500 people have Parkinson's

$\rightarrow$ PD has motor and non-motor symptoms

$\rightarrow$ PD progression is not linear and varies among individuals 


\section{PD traditional assessment}

It's not suitable for continuous monitoring

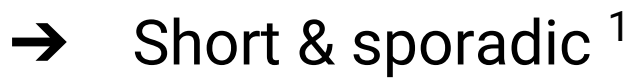

$\rightarrow$ Clinical expertise needed $^{2}$

$\rightarrow$ Resource consuming ${ }^{3}$

$\rightarrow$ Uncomfortable for patients ${ }^{4}$

$\rightarrow$ Symptoms vary during the day ${ }^{5}$

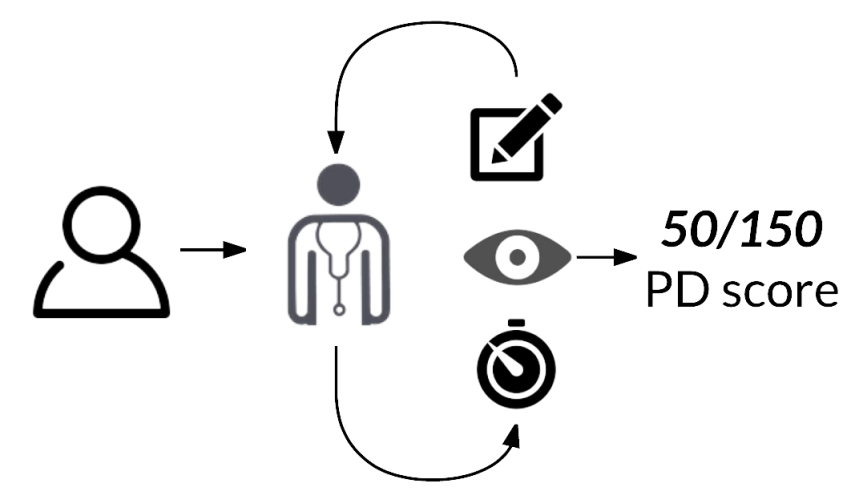




\section{Alternative assessment approaches}

$\rightarrow \quad$ Paper \& electronic diaries

$\rightarrow$ Electronic surveys

$\rightarrow$ Technology-supported:

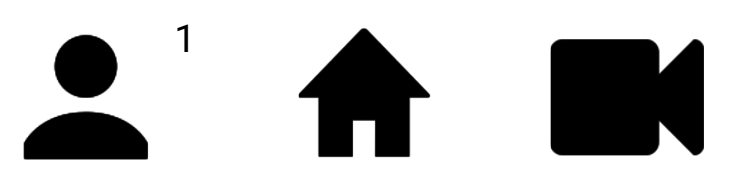

Personal Environmental Surveillance

${ }^{1}$ Wearables and/or smartphones measuring mostly tremor, slow movement, activities of daily living, gait and voice features. 


\section{Main research question}

Can PD be monitored via human behaviour inferred from smartphone collected data? 


\section{My approach}

Measure PD progression using proxies derived from heterogeneous data and human behaviour

$\rightarrow$ Longitudinal

$\rightarrow$ Non-disruptive

$\rightarrow$ Non-intrusive

$\rightarrow$ Naturalistic

$\rightarrow$ Multi-source

$\rightarrow$ Macro-scale

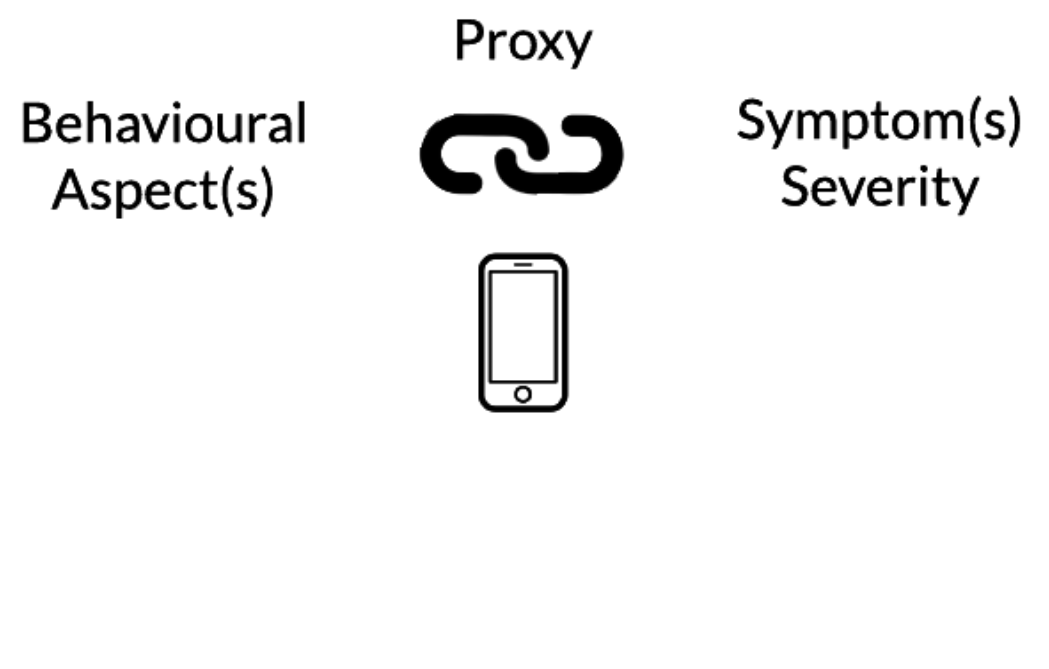




\section{Methodology}

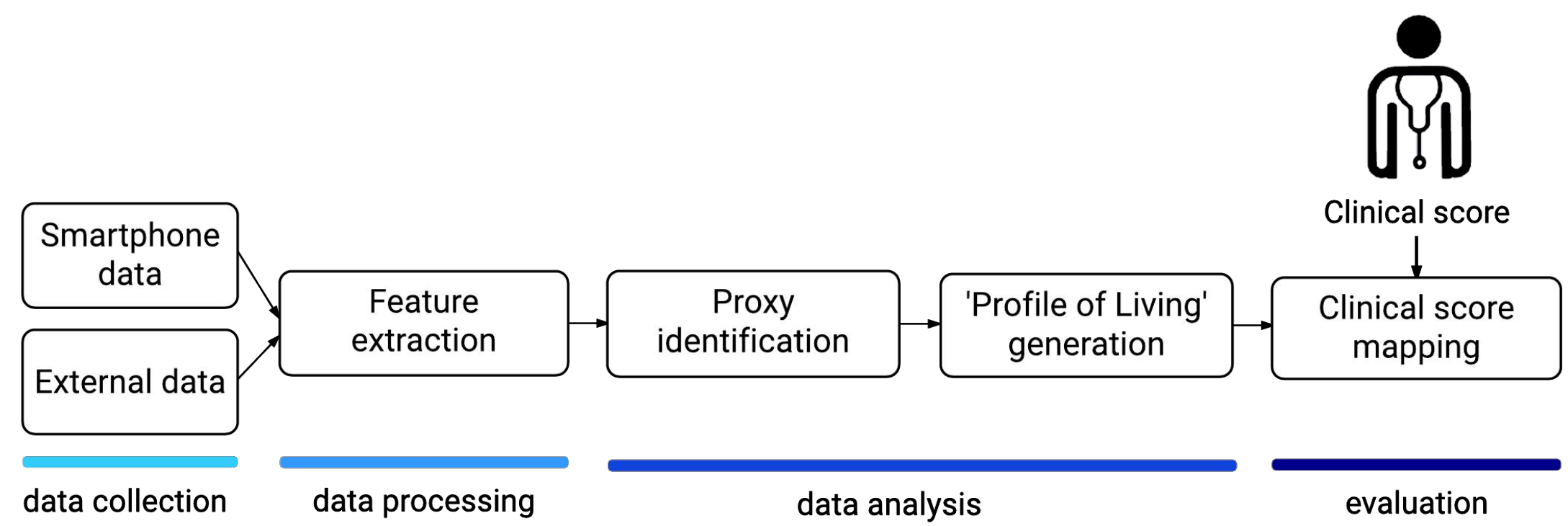




\section{Progress to date}

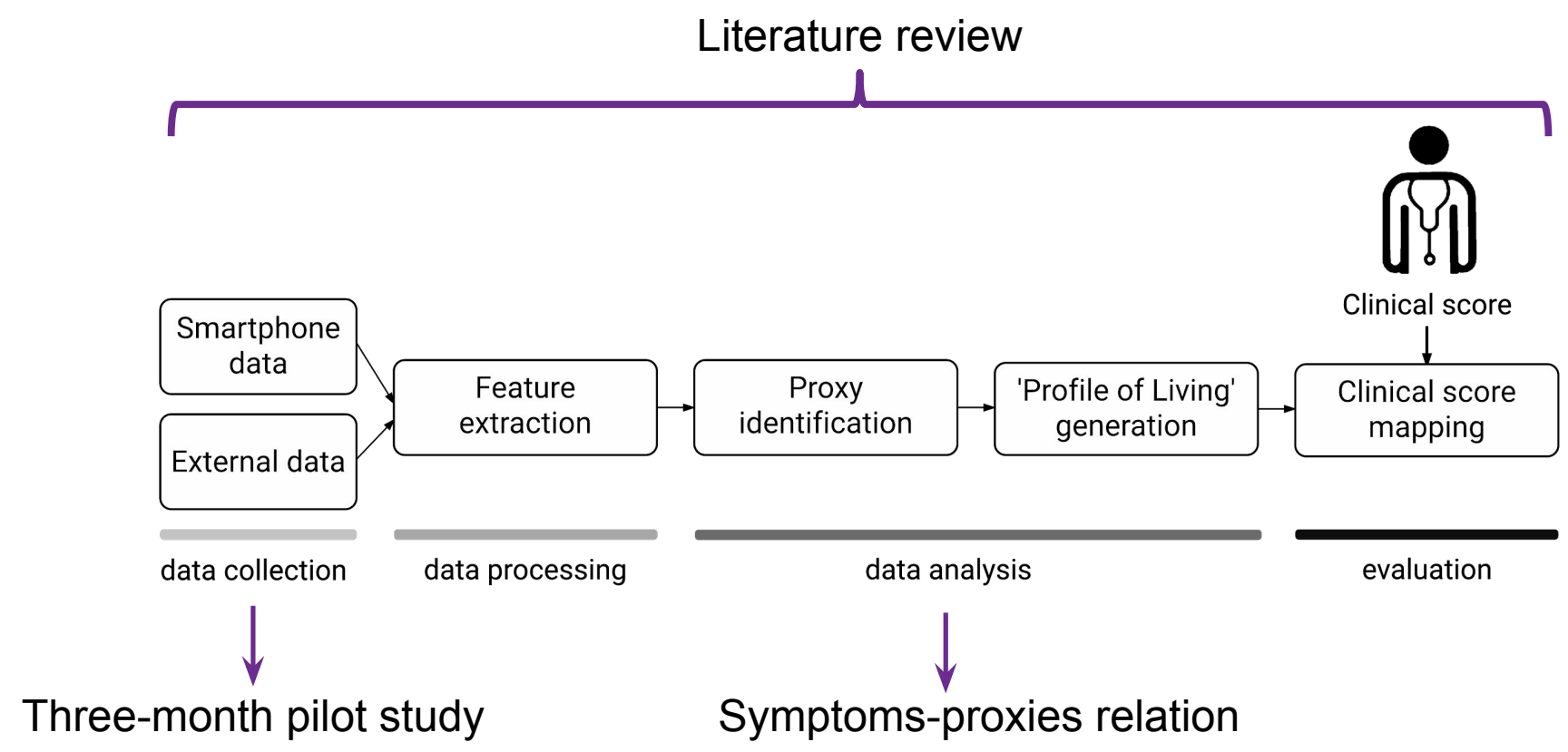

We are carrying out a one-year monitoring study 


\section{Pilot study}

Collected 29 data sources from two participants during three months

$\rightarrow$ Inertial data (accelerometer, gyro, rotation...)

$\rightarrow$ Communication data (calls, sms, bluetooth, wifi)

$\rightarrow$ Spatial data (GPS, cell towers, time zone, GIS)

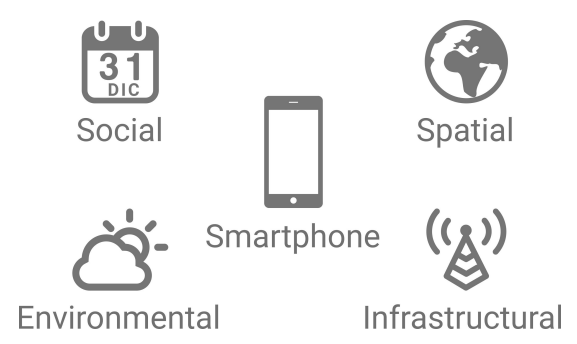

$\rightarrow$ Environmental data (weather, light, barometer, noise)

$\rightarrow$ Web \& Interaction data (used apps \& websites, touch gestures, keyboard types) 


\section{Data analysis}

Hypothetical Proxies
A. Typing patterns
B. Phone usage
C. Going up/down stairs
D. Indoors routine
E. Motor activities
F. Social patterns

\section{Profile of Living}

1. Individual baseline

2. Fluctuation measurement 


\section{Proxies}

A proxy has metrics. Metrics are of two types

$\rightarrow$ Direct

- Directly quantifiable. Single-source

$\rightarrow \quad$ Latent

- Need a tailored scale. Multi-source 


\section{For example}

\begin{tabular}{|l|l|l|}
\hline \multicolumn{1}{|c|}{ Proxy } & \multicolumn{1}{|c|}{ Direct Metrics } & \multicolumn{1}{|c|}{ Latent Metrics } \\
\hline Typing patterns & $\begin{array}{l}\text { Typing episodes (length, frequency) } \\
\text { Duration of halts between typing events } \\
\text { Typing duration for different word lengths }\end{array}$ & Typing context (location, time) \\
\hline Phone usage patterns & $\begin{array}{l}\text { Frequency of phone use } \\
\text { Visited websites } \\
\text { Touch gestures metrics (e.g. taps on target) }\end{array}$ & $\begin{array}{l}\text { Start of morning routine } \\
\text { Phone usage context (location, noise, time) } \\
\text { Website content analysis }\end{array}$ \\
\hline Going up/down stairs & $\begin{array}{l}\text { Frequency of episodes } \\
\text { Duration of episodes } \\
\text { Speed of episodes }\end{array}$ & Episodes' context (time of the day, week...) \\
\hline Social patterns & $\begin{array}{l}\text { Communication (calls and sms) } \\
\text { Applications used (whatsapp, email) } \\
\text { Location (commonly visited places) }\end{array}$ & $\begin{array}{l}\text { Time spent at work/home } \\
\text { Weekly routine } \\
\text { Individuals around participants }\end{array}$ \\
\hline
\end{tabular}




\section{Options for quantifying latent metrics}

$\rightarrow$ Regularity patterns

$\rightarrow$ Text mining techniques

$\rightarrow$ Bayesian and entropy based techniques

$\rightarrow$ Eigenvectors-like techniques

$\rightarrow$ Fuzzy logic

$\rightarrow$ Scanpath analysis from eye tracking 


\section{Profile of Living}

The proxy's metrics evolution is quantified using a PoL

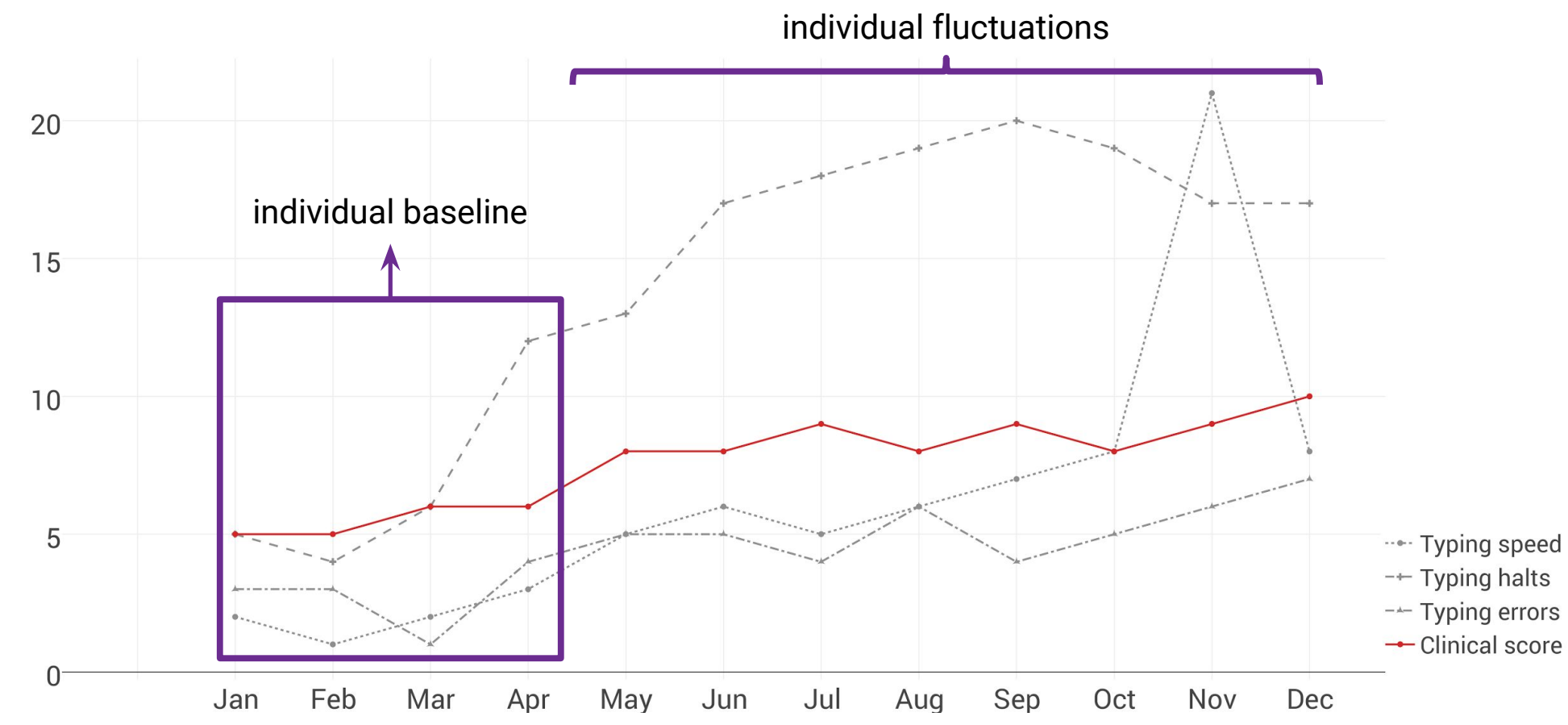




\section{Evaluation}

Monthly (adaptative) scoring of PD using different clinical scales

$\rightarrow \quad$ MDS-UPDRS (motor \& non-motor aspects of PD, widely use in research)

$\rightarrow$ Montreal Cognitive Assessment scale (MoCA)

$\rightarrow$ Geriatric Depression scale

$\rightarrow$ Activities of Daily Living scale

$\rightarrow$ Tech skills scale

$\rightarrow$ Self-reported evaluation of symptoms' impact in daily routine 


\section{Future work}

$\rightarrow$ Recruit more participants for the study

$\rightarrow$ Collect more data during the next 11 months

$\rightarrow$ Pick one proxy and explore its metrics

$\rightarrow \quad$ Evaluate the proxy using PD clinical scales 


\section{Interesting questions in my research}

$\rightarrow \quad$ How can touch interaction data be analysed to find a PD proxy?

$\rightarrow$ How to quantify latent metrics? (no labelled data)

$\rightarrow$ How to measure the PoL's baseline? Dynamically, statically?

$\rightarrow$ Are there any opportunities to leverage any new proxies?

$\rightarrow$ What is the best time granularity/behavioural abstraction for data analysis?

$\rightarrow$ What is the best frequency for ground truth observations? 


\title{
Thank you
}

Monitoring Parkinson's Disease Progression Using Behavioural Inferences, Mobile Devices and Web Technologies

Interaction Analysis and Modelling Lab

\author{
Julio Vega \\ julio.vega@manchester.ac.uk
}

